Bull. Korean Math. Soc. 50 (2013), No. 1, pp. 175-184

http://dx.doi.org/10.4134/BKMS.2013.50.1.175

\title{
ON THE ISOPERIMETRIC DEFICIT UPPER LIMIT
}

\author{
Jiazu Zhou, Lei Ma, And Wenxue Xu
}

\begin{abstract}
In this paper, the reverse Bonnesen style inequalities for convex domain in the Euclidean plane $\mathbb{R}^{2}$ are investigated. The Minkowski mixed convex set of two convex sets $K$ and $L$ is studied and some new geometric inequalities are obtained. From these inequalities obtained, some isoperimetric deficit upper limits, that is, the reverse Bonnesen style inequalities for convex domain $K$ are obtained. These isoperimetric deficit upper limits obtained are more fundamental than the known results of Bottema ([5]) and Pleijel ([22]).
\end{abstract}

\section{Introductions and preliminaries}

Perhaps the best known geometric inequality is the classical isoperimetric inequality. And its analytic proofs root back to centuries ago. One can find some simplified and beautiful proofs that lead to generalizations of higher dimensions and applications to other branches of mathematics (cf. [1], [6], [10], [11]-[17], [16], [20], [21], [24]-[25], [27], [29], [30], [31], [32], [34]-[35], [37], [39]).

The classical isoperimetric inequality says that: the circle is the only curve of constant perimeter enclosing the maximum area. This property is most precisely expressed in the following inequality:

$$
P^{2}-4 \pi A \geq 0,
$$

where $P$ and $A$ are, respectively, the perimeter of curve $\Gamma$ and the area $\Gamma$ encloses. The equality sign holds if and only if $\Gamma$ is a circle.

Let $K$ be a domain with the boundary composing of the simple curve $\partial K$ of perimeter $P_{K}$ and area $A_{K}$ in the Euclidean plane $\mathbb{R}^{2}$. The isoperimetric deficit of $K$ is defined as

$$
\Delta_{2}(K)=P_{K}^{2}-4 \pi A_{K} .
$$

The isoperimetric deficit $\Delta_{2}(K)$ measures the deficit between a domain $K$ and a disc of radius $\frac{P_{K}}{2 \pi}$. During the 1920 's, Bonnesen proved a series of inequalities

Received July 10, 2011; Revised February 1, 2012.

2010 Mathematics Subject Classification. Primary 52A10; Secondary 52A22.

Key words and phrases. convex domain, the Minkowski mixed area, the isoperimetric deficit upper limit, the Bonnesen style inequality, the reverse Bonnesen style inequality.

Supported in part by NSFC (grant number: 10971167).

(C)2013 The Korean Mathematical Society 
of the form

$$
\Delta_{2}(K) \geq B_{K}
$$

where the quantity $B_{K}$ is an invariant of geometric significance having the following basic properties:

1. $B_{K}$ is non-negative;

2. $B_{K}$ is vanish only when $K$ is a disc.

Many $B$ s are found in the last century and mathematicians are still working on those unknown invariants of geometric significance. An inequality of type (3) is called a Bonnesen style inequality. See [2], [3], [4], [6], [10], [16], [19], [21], [24], [25], [34] and [33] for more detailed references.

A set of points $K$ in the Euclidean space $\mathbb{R}^{n}$ is convex if for all $x, y \in K$ and $0 \leq \lambda \leq 1, \lambda x+(1-\lambda) y \in K$. The convex hull $K^{*}$ of a set of points $K$ in $\mathbb{R}^{n}$ is the intersection of all convex sets that contain $K$. A domain is a compact set with nonempty interiors. Since for any domain $K$ in $\mathbb{R}^{2}$, its convex hull $K^{*}$ increases the area $A^{*}$ and decreases the perimeter $P^{*}$. Then we have $P^{2}-4 \pi A \geq P^{* 2}-4 \pi A^{*}$, that is, $\Delta_{2}(K) \geq \Delta_{2}\left(K^{*}\right)$. Therefore the isoperimetric inequality and the Bonnesen style inequality are valid for all domains in $\mathbb{R}^{2}$ if these inequalities are valid for convex domains.

The following known inequality is so-called the Bonnesen isoperimetric inequality:

Proposition 1. Let $K$ be a convex domain of area $A_{K}$ and perimeter $P_{K}$. Let $r_{I}(K)$ and $r_{E}(K)$ be the radius of the maximum inscribed disc and the radius of the minimum circumscribed disc, respectively, of $K$. Then

$$
\Delta_{2}(K)=P_{K}^{2}-4 \pi A_{K} \geq \pi^{2}\left(r_{E}(K)-r_{I}(K)\right)^{2},
$$

where the equality holds if and only if $K$ is a disc.

When mathematicians are mainly interested in and focus on the lower bounds of the isoperimetric deficit, that is, the Bonnesen-type inequalities, there is another question: Is there invariant $U_{K}$ of geometric significance such that

$$
\Delta_{2}(K) \leq U_{K} ?
$$

That is, to find the reverse Bonnesen style inequality. We also expect that the upper bound be attained when $K$ is a disc. We are not aware of any general upper bound up today except for few results for special convex domains, that is, for oval domains ([5], [22], [25]).

Let $K$ be an oval domain in $\mathbb{R}^{2}$ with the continues radius of curvature $\rho$ of the boundary $\partial K$. Bottema (see [5], [25]) finds the following reverse Bonnesen isoperimetric inequality:

Proposition 2. Let $K$ be a convex domain of area $A_{K}$ and perimeter $P_{K}$ with the continuous radius of curvature $\rho$ of $\partial K$. Let $\rho_{m}$ and $\rho_{M}$ be the smallest and the greatest values, respectively, of $\rho$. Then

$$
\Delta_{2}(K)=P_{K}^{2}-4 \pi A_{K} \leq \pi^{2}\left(\rho_{M}-\rho_{m}\right)^{2} .
$$


The equality sign holds if and only if $\rho_{M}=\rho_{m}$, that is, $K$ is a disk.

Recently, the Bottema's inequality (5) has been generalized to the plane of constant curvature in [19].

Pleijel (see [22], [25]) has an improvement of Bottema's result as follows:

Proposition 3. Let $K$ be a convex domain of area $A_{K}$ and perimeter $P_{K}$ with the continuous curvature radius $\rho$ of $\partial K$. Let $\rho_{m}$ and $\rho_{M}$ be the smallest and the greatest values, respectively, of $\rho$. Then

$$
\Delta_{2}(K)=P_{K}^{2}-4 \pi A_{K} \leq \pi(4-\pi)\left(\rho_{M}-\rho_{m}\right)^{2} .
$$

The equality sign holds if and only if $K$ is a disc.

In this paper, we first investigate the Minkowski mixed area of two convex sets $K, L$ in the Euclidean plane $\mathbb{R}^{2}$ and obtain some geometric inequalities involving the mixed area $A_{K, L}$ of $K$ and $L$. By these inequalities obtained we derive some reverse Bonnesen style inequalities for a convex domain $K$. These reverse Bonnesen style inequalities, that is, the isoperimetric deficit upper bounds obtained, are invariants involving area $A_{K}$, circum length $P_{K}$, radius $r_{I}$ of the inscribed disc and the radius $r_{E}$ of the circumscribed disk of $K$. As we expected, those reverse Bonnesen style inequalities are held as equalities if and only if $K$ is a disc. These upper limits obtained are analogues of Bottema's (5) and Pleijel's (6). One of the main results is Theorem 3 that improves the isoperimetric deficit upper limit of Bokowski and Heil in [2].

\section{The mixed convex set of convex sets in $\mathbb{R}^{2}$}

A line $G$ in the Euclidean plane $\mathbb{R}^{2}$ can be determined by its distance $p$ from the origin $O$ and the angle $\phi$ of the normal with the $x$-axis. The line equation can be expressed as

$$
G(p, \phi): \quad x \cos \phi+y \sin \phi-p=0, \quad 0 \leq p<+\infty, \quad 0 \leq \phi \leq 2 \pi .
$$

If function $p=p(\phi)$ is of class $C^{2}$ and of periodic, then the envelope of the family of lines is:

$$
x=p \cos \phi-p^{\prime} \sin \phi . \quad y=p \sin \phi+p^{\prime} \cos \phi .
$$

If the envelope is the boundary $\partial K$ of a convex set $K$ and $O$ is an interior point of $K$, then $p=p(\phi)$ is called the support function of $K$ (or the support function of the convex curve $\partial K$ with reference to the origin $O$ ). The lines $(7)$ are called the support lines of $K$. Therefore we can prove that $p+p^{\prime \prime}>0$, and the arc-parameter is

$$
d s=\left(p+p^{\prime \prime}\right) d \phi .
$$

It is well-known that a necessary and sufficient condition that a function of period $2 \pi$ should be the support function of a convex set $K$ is that

$$
p(\phi)+p^{\prime \prime}(\phi)>0, \quad 0 \leq \phi<2 \pi .
$$


From the Cauchy's formula for convex sets, we have the perimeter of $K$ is

$$
P_{K}=\int_{0}^{2 \pi} p(\phi) d \phi
$$

and the area of a convex set $K$ can also be evaluated by its support function, that is,

$$
A_{K}=\frac{1}{2} \int_{\partial K} p d s=\frac{1}{2} \int_{0}^{2 \pi} p\left(p+p^{\prime \prime}\right) d \phi
$$

or

$$
A_{K}=\frac{1}{2} \int_{0}^{2 \pi}\left(p^{2}-p^{\prime 2}\right) d \phi
$$

Let $K, L$ be compact convex sets with, respectively, support functions $p_{K}$, $p_{L}$ assumed of class $C^{2}$. Then $p_{K}+p_{L}$ determines a convex set $M(K, L)$ called the mixed convex set of $K$ and $L$.

The perimeter of $M(K, L)$ is

$$
P_{M(K, L)}=P_{K}+P_{L},
$$

and the area of $M(K, L)$ is given by

$$
A_{M(K, L)}=A_{K}+A_{L}+2 A_{K, L} .
$$

The Minkowski mixed area of $K$ and $L$ is defined as

$$
A_{K, L}=\frac{1}{2} \int_{0}^{2 \pi}\left(p_{K} p_{L}-p_{K}^{\prime} p_{L}^{\prime}\right) d \phi .
$$

Integration by parts gives,

$$
A_{K, L}=\frac{1}{2} \int_{0}^{2 \pi} p_{K}\left(p_{L}+p_{L}^{\prime \prime}\right) d \phi=\frac{1}{2} \int_{0}^{2 \pi} p_{L}\left(p_{K}+p_{K}^{\prime \prime}\right) d \phi=A_{L, K} .
$$

Therefore we have the following results:

Lemma 1. For convex sets $K$ and $L, A_{K, L}$ is rigid invariant.

Lemma 2. For any convex set $K$,

$$
A_{K, K}=A_{K}
$$

Lemma 3. The mixed area $A_{K, L}$ is monotonic, that is, for convex sets $K, L_{1}$, $L_{2}$ such that $L_{1} \subset L_{2}$, then

$$
A_{K, L_{1}} \leq A_{K, L_{2}}
$$

Lemma 4. Let $K$ be a convex set. Then for the disc $B_{r}$ of radius $r$, we have

$$
A_{K, B_{r}}=\frac{r}{2} P_{K}
$$

Lemma 5 (Minkowski). Let $K$ and $L$ be convex sets. Then

$$
A_{K, L}^{2}-A_{K} A_{L} \geq 0,
$$

where equality holds if and only if $K$ and $L$ are homothetic. 
Let $K$ be a convex set and $B_{r}$ be disc of radius $r$. Then by Lemma 4 we have

$$
A_{K, B_{r}}^{2}-A_{K} A_{B_{r}}=\left(\frac{r}{2} P_{K}\right)^{2}-\pi r^{2} A_{K}=\frac{r^{2}}{4}\left(P_{K}^{2}-4 \pi A_{K}\right) .
$$

Then by Lemma 5 we immediately obtain the known classical isoperimetric inequality (1).

\section{The isoperimetric deficit upper limit for convex domains in $\mathbb{R}^{2}$}

Let $K$ be a convex domain of length $P_{K}$ and area $A_{K}$ in $\mathbb{R}^{2}$. Assume that $K$ encloses a maximum inscribed circle of radius $r_{I}$ and is circumscribed in a smallest circle of radius $r_{E}$. Then we have the following inequalities that will lead to our isoperimetric upper limits.

Theorem 1. Let $K$ be a convex domain in the Euclidean plane $\mathbb{R}^{2}$ and $d_{K}$ be the diameter of $K$. Then the invariants $P_{K}, A_{K}, r_{I}, r_{E}$ of $K$ satisfy the following inequalities:

$$
r_{I} \leq \frac{2 A_{K}}{P_{K}} \leq \sqrt{\frac{A_{K}}{\pi}} \leq \frac{P_{K}}{2 \pi} \leq \frac{d_{K}}{2} \leq r_{E} .
$$

Each equality sign holds if and only if $K$ is a disc.

Proof. Since the inequalities $\frac{2 A_{K}}{P_{K}} \leq \sqrt{\frac{A_{K}}{\pi}} \leq \frac{P_{K}}{2 \pi}$ are just the variant forms of the isoperimetric inequality. The inequality $\frac{P_{K}}{2 \pi} \leq \frac{d_{K}}{2} \leq r_{E}$ is known ([25], [24]). Therefore we just need to prove the first inequality $r_{I} \leq \frac{2 A_{K}}{P_{K}}$.

The inequality

$$
r_{I} \leq \frac{2 A_{K}}{P_{K}}
$$

comes immediately from Lemma 2, Lemma 3 and Lemma 4 . We complete the proof of Theorem 1.

Remark 1. The inequality (23) holds for all convex domains, with $C^{2}$-smooth or non $C^{2}$-smooth boundary ([36], [37]). Therefore all inequalities in (22) are valid for all convex domains, even for convex domains with non $C^{2}$-smooth boundaries.

Via inequalities

$$
r_{I} \leq \frac{2 A_{K}}{P_{K}} \leq \frac{P_{K}}{2 \pi} \leq r_{E}
$$

in (22), we have

$$
\frac{P_{K}}{2 \pi}-\frac{2 A_{K}}{P_{K}} \leq r_{E}-r_{I},
$$

that is,

$$
P_{K}^{2}-4 \pi A_{K} \leq 2 \pi P_{K}\left(r_{E}-r_{I}\right) .
$$

We have proved the following: 
Theorem 2. Let $K$ be a convex domain of perimeter $P_{K}$, and area $A_{K}$ in the Euclidean plane $\mathbb{R}^{2}$. Let $r_{I}$ and $r_{E}$ be, respectively, the in-radius and the circum-radius of $K$. Then we have the following reverse Bonnesen style inequality:

$$
\Delta_{2}(K)=P_{K}^{2}-4 \pi A_{K} \leq 2 \pi P_{K}\left(r_{E}-r_{I}\right),
$$

where the equality holds if and only if $K$ is a disc.

The inequality (24) is also obtained by Bokowski and Heil for convex domain $K$ with $C^{2}$-smooth boundary $\partial K$ (see [2]) by a different approach. And they state that the inequality is better than Favard's inequality (cf. page 83 in [4]) for the minimal circular annulus (minimalkreisring).

Via inequalities

$$
r_{I} \leq \frac{2 A_{K}}{P_{K}} \leq \frac{P_{K}}{2 \pi} \leq \frac{d_{K}}{2}
$$

from (22), we have

$$
\frac{P_{K}}{2 \pi}-\frac{2 A_{K}}{P_{K}} \leq \frac{d_{K}}{2}-r_{I}(K)
$$

that is,

$$
P_{K}^{2}-4 \pi A_{K} \leq 2 \pi P_{K}\left(\frac{d_{K}}{2}-r_{I}\right)
$$

We obtain the following isoperimetric deficit upper limit that is an improvement of Bokowski and Heil's inequality (24).

Theorem 3. Let $K$ be a convex domain of perimeter $P_{K}$ and area $A_{K}$ in the Euclidean plane $\mathbb{R}^{2}$. Let $r_{I}$ and $d_{K}$ be, respectively, the in-radius and the diameter of $K$. Then we have the following reverse Bonnesen style inequality:

$$
\Delta_{2}(K)=P_{K}^{2}-4 \pi A_{K} \leq 2 \pi P_{K}\left(\frac{d_{K}}{2}-r_{I}\right)
$$

where the equality holds if and only if $K$ is a disc.

From inequalities

$$
r_{I} \leq \frac{2 A_{K}}{P_{K}} \leq \sqrt{\frac{A_{K}}{\pi}} \leq r_{E}
$$

in (22), we have

$$
r_{I}^{2} \leq \frac{4 A_{K}^{2}}{P_{K}^{2}} \leq \frac{A_{K}}{\pi} \leq r_{E}^{2},
$$

and then

$$
\frac{A_{K}}{\pi}-\frac{4 A_{K}^{2}}{P_{K}^{2}} \leq r_{E}^{2}-r_{I}^{2} .
$$

Therefore we have

$$
P_{K}^{2}-4 \pi A_{K} \leq \frac{\pi P_{K}^{2}}{A_{K}}\left(r_{E}^{2}-r_{I}^{2}\right) .
$$


By inequalities

in $(22)$, we have

$$
r_{I} \leq \sqrt{\frac{A_{K}}{\pi}} \leq \frac{P_{K}}{2 \pi} \leq r_{E}
$$

and then

$$
r_{I}^{2} \leq \frac{A_{K}}{\pi} \leq \frac{P_{K}^{2}}{4 \pi^{2}} \leq r_{E}^{2}
$$

that is,

$$
\frac{P_{K}^{2}}{4 \pi^{2}}-\frac{A_{K}}{\pi} \leq r_{E}^{2}-r_{I}^{2}
$$

$$
P_{K}^{2}-4 \pi A_{K} \leq 4 \pi^{2}\left(r_{E}^{2}-r_{I}^{2}\right) .
$$

We have proved the following theorem.

Theorem 4. Let $K$ be a convex domain of perimeter $P_{K}$ and area $A_{K}$ in the Euclidean plane $\mathbb{R}^{2}$. Let $r_{I}$ and $r_{E}$ be, respectively, the in-radius and the circum-radius of $K$. Then we have the following reverse Bonnesen style inequalities:

$$
\begin{aligned}
& \Delta_{2}(K)=P_{K}^{2}-4 \pi A_{K} \leq \frac{\pi P_{K}^{2}}{A_{K}}\left(r_{E}^{2}-r_{I}^{2}\right) \\
& \Delta_{2}(K)=P_{K}^{2}-4 \pi A_{K} \leq 4 \pi^{2}\left(r_{E}^{2}-r_{I}^{2}\right) .
\end{aligned}
$$

Each equality sign holds if and only if $K$ is a disc.

Also by inequalities

we have:

$$
r_{I} \leq \frac{2 A_{K}}{P_{K}} \leq \frac{P_{K}}{2 \pi} \leq \frac{d_{K}}{2}
$$

Theorem 5. Let $K$ be a convex domain of the perimeter $P_{K}$ and area $A_{K}$ in the Euclidean plane $\mathbb{R}^{2}$. Let $r_{I}$ and $d_{K}$ be, respectively, the in-radius and the diameter of $K$. Then we have the following reverse Bonnesen style inequalities:

$$
\begin{aligned}
& \Delta_{2}(K)=P_{K}^{2}-4 \pi A_{K} \leq \frac{\pi P_{K}^{2}}{A_{K}}\left(\frac{d_{K}^{2}}{4}-r_{I}^{2}\right) ; \\
& \Delta_{2}(K)=P_{K}^{2}-4 \pi A_{K} \leq 4 \pi^{2}\left(\frac{d_{K}^{2}}{4}-r_{I}^{2}\right) .
\end{aligned}
$$

Each equality holds as an equality if and only if $K$ is a disc.

Remark 2. By the isoperimetric inequality (1) and inequalities (22) we have

$$
\begin{aligned}
2 \pi P_{K}\left(\frac{d_{K}}{2}-r_{I}\right) & \leq 2 \pi P_{K}\left(r_{E}-r_{I}\right) \leq 4 \pi^{2} r_{E}\left(r_{E}-r_{I}\right) \\
& \leq 4 \pi^{2}\left(r_{E}^{2}-r_{I}^{2}\right) \leq \frac{\pi P_{K}^{2}}{A_{K}}\left(r_{E}^{2}-r_{I}^{2}\right)
\end{aligned}
$$

Therefore the inequality (25), that is,

$$
\Delta_{2}(K)=P_{K}^{2}-4 \pi A_{K} \leq 2 \pi P_{K}\left(\frac{d_{K}}{2}-r_{I}\right)
$$


is the best isoperimetric deficit upper limit.

Remark 3. Our isoperimetric deficit upper limits do not assume $K$ is an oval domain, that is, we do not assume $K$ with the continuous radius of curvature $\rho$. Therefore those isoperimetric deficit upper limits obtained are more fundamental than Bottema and Pleijel's results.

Acknowledgements. The authors would like to thank the anonymous referees for helpful comments and suggestions that directly lead to great improvement of the original manuscript.

\section{References}

[1] T. F. Banchoff and W. F. Pohl, A generalization of the isoperimetric inequality, J. Differential Geometry 6 (1971/72), 175-192.

[2] J. Bokowski and E. Heil, Integral representation of quermassintegrals and Bonnesenstyle inequalities, Arch. Math. (Basel) 47 (1986), no. 1, 79-89.

[3] T. Bonnesen, Les probléms des isopérimétres et des isépiphanes, Gauthier-Villars, Paris, 1929.

[4] T. Bonnesen and W. Fenchel, Theorie der konvexen Köeper, 2nd ed., Berlin-HeidelbergNew York, 1974.

[5] O. Bottema, Eine obere Grenze für das isoperimetrische Defizit ebener Kurven, Nederl. Akad. Wetensch. Proc. A66 (1933), 442-446.

[6] Yu. D. Burago and V. A. Zalgaller, Geometric Inequalities, Springer-Verlag Berlin Heidelberg, 1988.

[7] V. Diskant, A generalization of Bonnesen's inequalities, Soviet Math. Dokl. 14 (1973), 1728-1731 (Transl. of Dokl. Akad. Nauk SSSR 213 (1973), 519-521).

[8] H. Flanders, A proof of Minkowski's inequality for convex curves, Amer. Math. Monthly 75 (1968), 581-593.

[9] E. Grinberg, S. Li, G. Zhang, and J. Zhou, Integral Geometry and Convexity, Proceedings of the International Conference, World Scientific, 2006.

[10] E. Grinberg, D. Ren, and J. Zhou, The symetric isoperimetric deficit and the containment problem in a plan of constant curvature, preprint.

[11] L. Gysin, The isoperimetric inequality for nonsimple closed curves, Proc. Amer. Math. Soc. 118 (1993), no. 1, 197-203.

[12] H. Hadwiger, Die isoperimetrische Ungleichung in Raum, Elemente der Math. 3 (1948), $25-38$.

[13] _ Vorlesungen über Inhalt, Oberfläche und Isoperimetrie, Springer, Berlin, 1957.

[14] G. Hardy, J. E. Littlewood, and G. Polya, Inequalities, Cambradge Univ. Press, Cambradge/New York, 1952.

[15] R. Howard, The sharp Sobolev inequality and the Banchoff-Pohl inequality on surfaces, Proc. Amer. Math. Soc. 126 (1998), no. 9, 2779-2787.

[16] W. Y. Hsiang, An elementary proof of the isoperimetric problem, Chinese Ann. Math. Ser. A 23 (2002), no. 1, 7-12.

[17] C. C. Hsiung, Isoperimetric inequalities for two-dimensional Riemannian manifolds with boundary, Ann. of Math. 73 (1961), no. 2, 213-220.

[18] $\mathrm{H} . \mathrm{Ku}, \mathrm{M} . \mathrm{Ku}$, and $\mathrm{X}$. Zhang, Isoperimetric inequalities on surfaces of constant curvature, Canad. J. Math. 49 (1997), no. 6, 1162-1187.

[19] M. Li and J. Zhou, An upper limit for the isoperimetric deficit of convex set in a plane of constant curvature, Sci. in China 53 (2010), no. 8, 1941-1946.

[20] R. Osserman, The isoperimetric inequality, Bull. Amer. Math. Soc. 84 (1978), no. 6, $1182-1238$. 
[21] $\_$, Bonnesen-style isoperimetric inequality, Amer. Math. Monthly 86 (1979), no. $1,1-29$.

[22] A. Pleijel, On konvexa kurvor, Nordisk Math. Tidskr. 3 (1955), 57-64.

[23] G. Polya and G. Szego, Isoperimetric inequalities in mathematical physics, Annals of Mathematics Studies, no. 27, Princeton University Press, Princeton, N. J., 1951.

[24] D. Ren, Topics in Integral Geometry, World Scientific, Sigapore, 1994.

[25] L. A. Santaló, Integral Geometry and Geometric Probability, Reading, MA: AddisonWesley, 1976.

[26] R. Schneider, Convex Bodies: The Brunn-Minkowski Theory, Cambridge Univ. Press, Cambridge, 1993.

[27] E. Teufel, A generalization of the isoperimetric inequality in the hyperbolic plane, Arch. Math. 57 (1991), no. 5, 508-513.

[28] - Isoperimetric inequalities for closed curves in spaces of constant curvature, Results Math. 22 (1992), no. 1-2, 622-630.

[29] J. L. Weiner, A generalization of the isoperimetric inequality on the 2-sphere, Indiana Univ. Math. J. 24 (1974), 243-248.

[30] - Isoperimetric inequalities for immersed closed spherical curves, Proc. Amer. Math. Soc. 120 (1994), no. 2, 501-506.

[31] S. T. Yau, Isoperimetric constants and the first eigenvalue of a compact Riemannian manifold, Ann. Sci. Ec. Norm. Super. Paris 8 (1975), no. 4, 487-507.

[32] G. Zhang and J. Zhou, Containment measures in integral geometry, Integral geometry and convexity, 153-168, World Sci. Publ., Hackensack, NJ, 2006.

[33] J. Zhou, On Bonnesen-type inequalities, Acta. Math. Sinica, Chinese Series 50 (2007), no. 6, 1397-1402.

[34] J. Zhou and F. Chen, The Bonnesen-type inequalities in a plane of constant curvature, J. Korean Math. Soc. 44 (2007), no. 6, 1363-1372.

[35] J. Zhou, Y. Du, and F. Cheng, Some Bonnesen-style inequalities for higher dimensions, to appear in Acta. Math. Sinica.

[36] J. Zhou and L. Ma, The discrete isoperimetric deficit upper bound, preprint.

[37] J. Zhou and D. Ren, Geometric inequalities from the viewpoint of integral geometry, Acta Math. Sci. Ser. A Chin. Ed. 30 (2010), no. 5, 1322-1339.

[38] J. Zhou, Y. Xia, and C. Zeng, Some new Bonnesen-style inequalities, J. Korean Math. Soc. 48 (2011), no. 2, 421-430.

[39] C. Zeng, J. Zhou, and S. Yue, The symmetric mixed isoperimetric inequality of two planar convex domains, Acta Math. Sinica 55 (2012), no. 3, 355-362.

JIAZU ZHOU

School of Mathematics and Statistics

SOUTHWEST UNIVERSITY

Chongqing, 400715, P. R. China

AND

Southeast Guizhou Vocational College of Technology for Nationalities

Kaili, Guizhou 556000, P. R. China

E-mail address: zhoujz@swu.edu.cn

LEI MA

School of Mathematics and Statistics

SOUTHWEST UNIVERSITY

Chongqing, 400715, P. R. China

E-mail address: maleiyou@swu.edu.cn 
Wenxue Xu

School of Mathematics and Statistics

SOUTHWEST University

ChongQIng, 400715, P. R. China

E-mail address: wenxxu@swu.edu.cn 